\title{
Production and decay of single heavy spin-3/2 leptons in high energy electron-positron collisions
}

\author{
F. M. L. Almeida Jr., J. H. Lopes, \\ J. A. Martins Simões and A. J. Ramalho \\ Instituto de Física \\ Universidade Federal do Rio de Janeiro \\ Ilha do Fundão, Rio de Janeiro \\ BR-21945-970, RJ, Brazil
}

\begin{abstract}
We discuss the possibility of detecting heavy spin-3/2 leptons at LEP2 and NLC energies. The angular distributions of primary ordinary leptons are shown to be a good measure to discriminate between a heavy spin-3/2 lepton and a similar nonstandard spin-1/2 signal.

PACS 12.60.-i, 14.60.Hi
\end{abstract}


There is now an extensive amount of data that confirm the standard model as the theory of fundamental interactions. However, since it depends on a relatively large number of parameters adjusted by hand, there is an expectation that some extended theory will provide a deeper understanding of nature. As yet none of the theoretical proposals has achieved this goal. On the experimental side, a lot of effort has been put on the search for new particles, which could shed some light on the physics beyond the standard model. In this paper we study the production and decay of possible heavy spin-3/2 leptons at LEP2 energy $\sqrt{s}=190 \mathrm{GeV}$ and at a future new linear collider (NLC) at $\sqrt{s}=500 \mathrm{GeV}$.

The motivation for spin-3/2 leptons comes from at least three scenarios. In composite models ordinary spin- $1 / 2$ leptons and quarks are thought to be formed by more fundamental fermions. If this hypothesis is correct, then excited spin-3/2 states are expected to exist [1]. Composite fermions in enlarged groups ( relative to the standard group) also imply spin-3/2 fermions [2]. In supergravity gauge theories [3] the spin-3/2 gravitino is the fermionic counterpart to the gravitational field. More recently, spin-3/2 fields appear in a quaternionic quantum mechanical model[4] for composite leptons and quarks. Unfortunately, there exists no complete self-consistent theory of interacting spin-3/2 fields. At the classical level there is the problem of faster than light propagating modes [5]. The minimal electromagnetic coupling for pair production of spin-3/2 leptons violates unitarity just above threshold [6]. These problems make difficult a comparison of theoretical predictions for spin-3/2 objects with any possible "new physics" experimental signal. Some time ago it was pointed out [1] that some of these difficulties could be ameliorated for the coupling of spin-3/2 fermions to massive electroweak gauge bosons. Although this hypothesis is not a solution of the problems concerning spin-3/2 fields, one expects that it will allow that reliable phenomenological estimates be made, in the same spirit of the effective Fermi theory for weak interactions. The heavy gauge boson propagator can act as an effective form factor, and one can compute production cross sections for spin-3/2 fermions which violate unitarity in kinematical regions well above thresholds, as in the old Fermi model. There are some theoretical arguments for a high spin-3/2 mass. The well known Weinberg-Witten theorems [7] leave open the possibility of massive spin-3/2 states at higher energy scales. This is supported, to some extent, by a recent analysis of the LEP1 data carried out by Montero and Pleitez [8], which implies the bound $M_{3 / 2}>M_{Z}$. 
We are then lead to study massive spin-3/2 fermions with masses above the $\mathrm{Z}$ mass.

The phenomenology of spin-3/2 lepton production has been studied for electron-positron collisions [1], hadronic reactions [9] and more recently, for the electron-proton collider HERA [10] and the $e^{-} \gamma$ mode of the next generation of electron-positron colliders [11]. In this paper we turn our attention to the production and decay of a single spin-3/2 lepton in $e^{+} e^{-}$collisions at LEP2 and NLC energies. Spin-3/2 lepton pair production is also possible, but it is bounded by $M_{3 / 2}<\sqrt{s} / 2$, whereas single spin-3/2 lepton production can reach masses as high as $\sqrt{s}$. We compare our results for spin-3/2 with the analogous heavy spin- $1 / 2$ case. A detailed analysis of the production process of single heavy spin-1/2 leptons was carried out in reference [12, in the framework of different nonstandard electroweak models, and the model dependence of unpolarized production cross sections and distributions was found to be weak.

In order to reduce the model dependence on spin-3/2 couplings, we consider the following phenomenological currents:

$$
\begin{gathered}
J_{1}^{\mu}=e \bar{u}^{\mu}(p, 3 / 2)\left(c_{1 V}-c_{1 A} \gamma^{5}\right) u(k, 1 / 2) \\
J_{2}^{\mu}=\frac{e}{\Lambda} \bar{u}^{\lambda}(p, 3 / 2) q_{\lambda} \gamma^{\mu}\left(c_{2 V}-c_{2 A} \gamma^{5}\right) u(k, 1 / 2),
\end{gathered}
$$

where $\bar{u}^{\mu}(p, 3 / 2)$ represents a Rarita-Schwinger vector spinor, $q^{\mu}=p^{\mu}-k^{\mu}$ and $\Lambda$ is a mass scale that sets the strength of the interaction. We assume that the mixing between ordinary spin-1/2 leptons and heavy spin-3/2 leptons occurs only within the same lepton family, with conservation of lepton number. A more general classification of operators with dimension up to six, involving spin-3/2 fields is given by Burges and Schnitzer [13].

A spin-3/2 heavy lepton whose mass exceeds the experimental lower bound $M_{3 / 2}>M_{Z}$ generally decays via a two-body process. The widths of the spin-3/2 leptons, according to currents $J_{1}$ and $J_{2}$, are given by

$$
\Gamma^{(1)}=\frac{\alpha}{48}\left(c_{1 V}^{2}+c_{1 A}^{2}\right) M_{3 / 2} \frac{(1-\kappa)^{2}}{\kappa}\left(1+10 \kappa+\kappa^{2}\right)
$$

and

$$
\Gamma^{(2)}=\frac{\alpha}{48}\left(c_{2 V}^{2}+c_{2 A}^{2}\right) M_{3 / 2}\left(\frac{M_{3 / 2}}{\Lambda}\right)^{2} \frac{(1-\kappa)^{4}}{\kappa}(1+2 \kappa),
$$


where $\kappa=\left(M_{3 / 2} / M_{B}\right)^{2}$ and $M_{B}$ stands for the vector boson (W or Z) mass. For $\Lambda=M_{3 / 2}$ and coupling constants of the order of one, typical widths are then in the range $0.1-10 \mathrm{MeV}$, showing that the direct observation of these leptons is a hopeless task. The branching ratios for two-body decays are given in table 1 . For the charged spin-3/2 lepton the most favored signature will be one ordinary charged lepton (primary lepton) in one hemisphere and a $\mathrm{W}$ boson and a missing $\nu$ in the other. For the neutral case the dominant event will have one missing neutrino in one hemisphere, along with a $\mathrm{W}$ boson and charged ordinary lepton in the opposite hemisphere.

It is well known that there is no theoretical prediction on the absolute value of the total cross section for spin-3/2 production. We have then normalized the total cross section to the upper bound on similar spin- $1 / 2$ production [12. In Fig.1 we show the Feynman diagrams for single spin-3/2 lepton production. The explicit calculation of these diagrams leads to

$$
\begin{aligned}
\frac{d \sigma^{(1)}}{d x}= & \frac{\pi}{12} \alpha^{2} \frac{(1-\eta)^{2}}{M_{3 / 2}^{2}}\left\{f^{(s)} A_{1}\left[8 \eta+(1-\eta)^{2}\left(1-x^{2}\right)-4 B_{1} \eta x\right]\right. \\
& +f^{(t)}\left[A_{1}(1+\eta)(1+2 \eta)+B_{1} \eta(3-\eta)-2\left(A_{1}+B_{1}\right) \eta x\right. \\
& \left.\quad-(1-\eta)\left(A_{1}(1-2 \eta)+B_{1} \eta\right) x^{2}\right] \\
& \left.+f^{(s t)}\left(A_{1}+B_{1}\right)\left[4 \eta+(1-x)\left(4 \eta+(1-\eta)^{2}(1+x)\right)\right]\right\} \\
\frac{d \sigma^{(2)}}{d x}= & \frac{\pi}{96} \alpha^{2} \frac{(1-\eta)^{2}}{\eta \Lambda^{2}}\left\{8 f^{(s)}(1-\eta)^{2}\left[A_{2}\left(1+\eta+(1-\eta) x^{2}\right)+2 B_{2} x\right]\right. \\
& +f^{(t)}[1+\eta-(1-\eta) x]^{2} \\
& \times\left[(5+\eta) A_{2}-(3-\eta) B_{2}+\left(A_{2}+B_{2}\right)\left(2 x+(1-\eta) x^{2}\right)\right] \\
& -4 f^{(s t)}(1-\eta)\left(A_{2}+B_{2}\right)(1+x) \\
& \times[1+\eta+(1-\eta) x][1-\eta-(1+\eta) x]\}
\end{aligned}
$$

In the expressions above, $x=\cos \theta$, where $\theta$ is the angle of the primary lepton with respect to the incoming electron, and the coupling constants $g_{i V}$ and $g_{i A}$ refer to standard-model vertices of the form $-i e \gamma_{\mu}\left(g_{i V}-g_{i A} \gamma_{5}\right)$. In 
addition,

$$
\begin{gathered}
\eta=M_{3 / 2}^{2} / s, \\
f^{(s)}=\frac{s^{2}}{\left(s-M_{Z}^{2}\right)^{2}+\left(\Gamma_{Z} M_{Z}\right)^{2}}, \\
f^{(t)}=\frac{s^{2}}{\left(t-M_{Z}^{2}\right)^{2}}, \\
f^{(s t)}=f^{(s)} \frac{\left(s-M_{Z}^{2}\right)}{\left(t-M_{Z}^{2}\right)}, \\
A_{i}=\left(c_{i V}^{2}+c_{i A}^{2}\right)\left(g_{i V}^{2}+g_{i A}^{2}\right)
\end{gathered}
$$

and

$$
B_{i}=4 c_{i V} c_{i A} g_{i V} g_{i A},(i=1,2) .
$$

Fig. 2 shows the production cross sections for charged spin-3/2 leptons, both at $\sqrt{s}=190 \mathrm{GeV}$ and $\sqrt{s}=500 \mathrm{GeV}$. For the sake of comparison, the cross section for the production of a heavy spin-1/2 lepton is also shown for a model with mixing angles equal to $\theta_{i}=0.1$. Assuming that LEP2 and NLC luminosities will take on the conservative yearly values of $150 \mathrm{pb}^{-1}$ and $10 \mathrm{fb}^{-1}$ respectively, the production rate of a $120 \mathrm{GeV}$ spin-3/2 lepton is found to be around 4 events per year at LEP2 but may reach 700 events per year at NLC. The energy dependence of the total cross sections is displayed in Fig.3. The curves grow steeply with the center of mass energy, which suggests the breakdown of unitarity. As expected, this is more severe in the case of the higher-dimension operator $J_{2}$. However, this violation of unitarity occurs for energies far above the production threshold.

Clearly, it would be difficult to separate an eventual spin-3/2 heavy lepton from a corresponding spin- $1 / 2$ signal only on the basis of total cross sections. Therefore it is natural to investigate which distributions are useful to determine the spin of nonstandard heavy leptons, and/or to distinguish the phenomenological currents $J_{1}$ and $J_{2}$. The results of this study are summed up in Figs.4-6, which show the normalized angular distributions of the charged ordinary lepton that accompanies the charged heavy lepton. In Fig. 4 the structure of the phenomenological currents $J_{1}$ and $J_{2}$ was assumed to be $V \pm A, \mathrm{~V}$ or $\mathrm{A}$. One can see that there is no significant dependence on the coupling constants $c_{i V}$ and $c_{i A}$. In figs. 5-6 we compare the spin-1/2 and spin- $3 / 2$ cases. The primary charged lepton associated with the heavy 
spin-1/2 charged lepton is produced mostly in the forward hemisphere, especially at NLC energies. As a matter of fact, this behavior is reproduced in the case of spin-3/2 current $J_{1}$ when the lepton mass is large enough, but it may still be possible to distinguish the two angular distributions if polarized $e^{+} e^{-}$beams are employed, along the same lines as suggested in [12]. If heavy spin-3/2 lepton production is correctly described by current $J_{2}$, however, the probabilities of detecting the primary lepton in each hemisphere are roughly the same at LEP2 energies. Although this is no longer true at NLC energies, the normalized angular distribution which corresponds to current $J_{2}$ remains different from the other two.

In conclusion, we have shown that it is feasible to search for new spin$3 / 2$ heavy leptons at LEP2 and NLC colliders, and that in most cases their experimental signals can be discriminated from similar spin-1/2 effects.

Acknowledgments: This work was partially supported by CNPq, CAPES, FUJB, FAPERJ and FINEP. 


\begin{tabular}{lcc}
\hline$L_{3 / 2}^{-} \longrightarrow\left(l^{-} \nu_{l}\right) \nu$ & $L_{3 / 2}^{0} \longrightarrow\left(l^{+} \nu_{l}\right) e^{-}$ & 8 \\
\hline$L_{3 / 2}^{-} \longrightarrow($ hadrons $) \nu$ & $L_{3 / 2}^{0} \longrightarrow($ hadrons $) l^{-}$ & 50 \\
\hline$L_{3 / 2}^{-} \longrightarrow($ invisible $) e^{-}$ & $L_{3 / 2}^{0} \longrightarrow$ invisible & 5 \\
\hline$L_{3 / 2}^{-} \longrightarrow\left(l^{+} l^{-}\right) e^{-}$ & $L_{3 / 2}^{0} \longrightarrow\left(l^{+} l^{-}\right) \nu_{L}$ & 3 \\
\hline$L_{3 / 2}^{-} \longrightarrow($ hadrons $) e^{-}$ & $L_{3 / 2}^{0} \longrightarrow($ hadrons $) \nu_{L}$ & 18 \\
\hline
\end{tabular}

Table 1:Branching ratios (\%) for the two-body decays of heavy spin-3/2 leptons. The parentheses show the products of the $W$ (or $Z$ ) boson decay. 


\section{References}

[1] J. Leite Lopes, J. A. Martins Simões and D. Spehler, Phys. Lett. B94 (1980) 367; Phys. Rev. D23 (1981) 797; D25 (1982) 1854.

[2] Y. Tosa and R. E. Marshak, Phys. Rev. D32 (1985) 774.

[3] P. van Nieuwenhuizen, Phys. Rep. 68 (1981) 189, and references therein.

[4] S. Adler, Phys. Lett. B332 (1994) 358.

[5] G. Velo and D. Zwanziger, Phys. Rev. 186 (1969) 267.

[6] J. G. Taylor, Phys. Lett. 90B (1980) 143.

[7] S. Weinberg and E. Witten, Phys. Lett. B96 (1980) 59.

[8] J. C. Montero and V. Pleitez, Phys. Lett. B321 (1994) 267.

[9] D. Spehler, O. J. P. Eboli, G. C. Marques, S. F. Novaes and A. A. Natale, Phys. Rev. D36 (1987) 1358 ; J. Kuhn and P. Zerwas, Phys. Lett. 147B (1984) 189.

[10] F. M. L. Almeida Jr., J. A. Martins Simões and A. J. Ramalho, Nucl. Phys. B397 (1993) 502.

[11] O. J. P. Éboli, E. M. Gregores, J. C. Montero, S. F. Novaes and D. Spehler, IFT preprint IFT-P.018/95.

[12] F. M. L. Almeida Jr., J. H. Lopes, J. A. Martins Simões, P. Queiroz Filho and A. J. Ramalho, Phys. Rev. D51 (1995) 5990.

[13] C. J. C. Burges and H. J. Schnitzer, Nucl. Phys. B228 (1983) 464; Phys. Lett. 134B (1984) 329. 


\section{Figure captions}

Fig.1 The lowest order Feynman diagrams for the process $e^{+} e^{-} \longrightarrow$ $e^{-}\left(\nu_{e}\right) L^{+}\left(\bar{L}^{0}\right)$.

Fig.2 (a) Cross sections for single production of heavy spin-3/2 leptons at $\sqrt{s}=190 \mathrm{GeV}$. The cross section for a nonstandard spin- $1 / 2$ lepton of a vector doublet model is shown for comparison. (b) Same as (a), but at $\sqrt{s}=500 \mathrm{GeV}$.

Fig.3 Total cross sections as functions of the center of mass energy, for input masses $M_{L}=120 \mathrm{GeV}$ and $M_{L}=400 \mathrm{GeV}$.

Fig.4 Normalized angular distributions of the primary lepton for different couplings $(V \pm A, \operatorname{Vor} A)$ in spin-3/2 currents $J_{1}$ and $J_{2}$.

Fig.5 (a) Normalized angular distribution of the primary lepton in heavy spin-3/2 lepton single production, at LEP2 energies and for an input mass $M_{L}=120 \mathrm{GeV}$. The corresponding distribution for the case of heavy spin-1/2 lepton production is also shown. (b) Same as (a), but for an input mass $M_{L}=160 \mathrm{GeV}$.

Fig.6 (a) Normalized angular distribution of the primary lepton in heavy spin-3/2 lepton single production, at NLC energies and for an input mass $M_{L}=250 \mathrm{GeV}$. The corresponding distribution for the case of heavy spin-1/2 lepton production is also shown. (b) Same as (a), but for an input mass $M_{L}=400 \mathrm{GeV}$. 